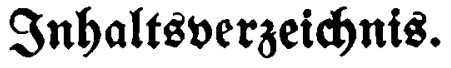

\section{Bürgerliđes Gejegbu何.}

\section{Erites Bud. Allgemetuer Teil.}

Erfter Mbidnitt. Berfonen

Gelte $\$ 8$

Erfter Titel. Ratürlide Berionen: : 1 1-20

3roeiter Titel. Suriftifhe Berfonen. 5

I. Bereine . . 5

1. Arlgemeine Boridriften . . . 5

2. Eingetragene Bereine . . . . 13

II. Stiftungen ........ 19

III. Эuriitifige ßerfonen bes öffentliden Redtes

$21-54$
$55-79$
$80-88$

\begin{tabular}{l|r}
24 & 89
\end{tabular}

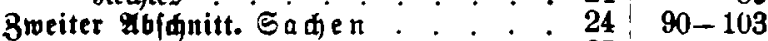

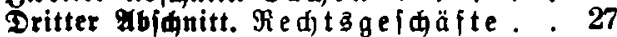

E r f́t ex Titel. Bejdäftşähigleit . . . 27 104-115

Breiter Titel. Willengertlärung . . $30116-144$

Ditter Titel. Retrag . . . 36 145-157

Bierter Titel. Bebingung. Beitbeftimmung

Fünfter Titel. Bertretung. Botlmadi

Sedfiter Titel. Eittoilligung. Beneh-

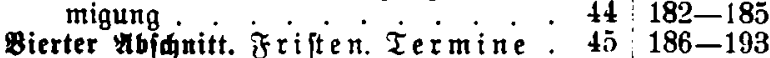

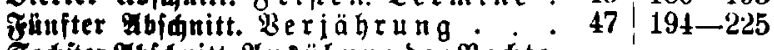

Sediter $\mathfrak{A b f d n i t t . ~} \mathfrak{A} u g u ̈ b u n g$ ber $\mathfrak{A}$ edte.

Selbftorteidigung. Selbfthilfe 5o $226-231$

Siebenter Tbinnitt. Siderheitsleifung $56,232-240$

\section{3weites Buक. Pedt ber Equlbberbaltuifie.}

Criter Mbiduitt. $\Im$ rhalt ber SduIb. berhältniffe... . . . . . 59

Eriter Titel. Berpfliditung zur \&eiftung $59 \mid 241-292$

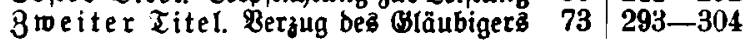


3weiter abidnnitt. Squlbverbăltniffe a us Berträgen. . . . . . 75

Eriter Titel. Begrünbung. Inbalt bes Bertrags

8 weiter $\mathfrak{T}$ it el. Begenfeitiger Bertrag Dritter ritel. Berpreden ber Leiftung an einen Dritten

Bierter Titel. Draufgabe. Bertraga: itrafe

Fünfter Titel. Rüdtritt

Dritter abidnitt. Erlöf由en ber S中ulb. verbätnilje.

Erfter Titel. Erfüllung.

Preiter Titel Sinterlegung....

Srelter Litel. Sinterlegung...9

Dritter Iitel. Oufrednung . . . 9

Bierter Titel. Erlas $\quad . \quad . \quad . \quad .99$

Bierter gbfdnitt. ub bertragung ber Forberung

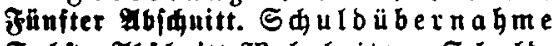

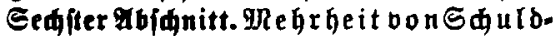
neтก นnd Bläubigern.

Siebenter $\mathfrak{A b}$ idnitt. Einzelne Gqulb.

berhältrifle........ . 105

Eriter Titel Rauf. Taujd... . 100̃

I. Allgemeine Boridriften . . . . 105

II. Gewährleiftung toegen Mängel ber Sadje $105 \quad 433-458$

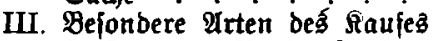

1. Rauf nad $\mathfrak{P r o b e}$. Sauf auf $\mathfrak{R}$ robe

2. Miebertauf . . . . . . 134

3. Bortauf . . . . . . . 136

TV. Tauid $: 137$

3weiter Titel. Sdentung . . . 138

Dritter Titel. Miete. Radt . . . 144

I. Miete . . . . . . . . . . 144

II. Radt . . . . . . . 155

Bierter Titel. Leibe . . . . . . 159

Fünfter Titel. Darłehen $: . . \quad .161$

Gediter Titel. Dienjtuertrag . . . 162

$305-319$

$320-327$

$328-335$

$336-345$

$346-361$

362-371

372-386

387-396 397

$398-413$

414- 419

420-432

$459-493$

494-496

497-503

504-514 515

516-534

535-580

$581-597$

598-606

607-610

611-630 
Ingalt Bberzetфuls.

VII

Gelle

Giebenter Titel. Berbertrag . . 168

צ́bter Titel. Mällerbertrag . . . 180

Reunter Titel. Ğalobung . . . 181

Behnter Titel. 2uftrag. . . . 182

Elfter Titel. Beídäftgührung ohne Euftrag

Boolfter Titel Berwahrung

Dreizeh nter Titel. Einbringung oon Sachen bei Baftwirten . . . . 189

Bierzebnter Titel. Bejellidaft . . 191

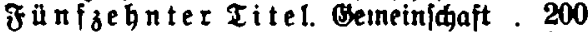

Se由zebnter Titel. Seibrente . . 204

Siebzehnter Titel. Spiel,

भatzebnter Titel. Bürgídaft . . 205

Reunzehnter Titel. Bergleid . 208

3 manzigiter Titel. Sdjulbverfpreden. Squlbanertenntnis... .209

(Finunbzronzigfter Titel. Antweifung 209

3 wei unbztwanzigiter Titel. Sd)ulb. veridreibung auj ben Эnhaber. . . 211

Dreiunbzroarzigiter Titel. Borlegung von Sahen

Bierunbztoanjiglter Titel. ungeredtfertigte Bereiderung . . . 217

Fünfunbzwanzigfter Titel. Unerlaubte sanblungen . . . . . 220

\section{Dritte Ou两. Oápenreht.}

Erfter tbidnitt. Befit. . . . . . 229 3wciter Mbidnitt. MIlgemeine 3 or: icriften über Medte an (Grund. it ü de $\pi$

Dritter Mbidntt. Figentum . . . 239

Eriter Titel. Tnhalt bes Eigentums 239

3 weiter Titel. Erwerb und Berluft Des Eigentums an Brunbitüđen. . 245

Dritter Titel. Erwerb unb Berlult beş Eigentums an betoeglidjen હađen 246 I. Utbertragung . . . . . . . 246

\begin{tabular}{|r} 
VII \\
88 \\
\hline $631-651$ \\
$652-656$ \\
$657-661$ \\
$662-676$ \\
$677-687$ \\
$688-700$ \\
$701-704$ \\
$705-740$ \\
$741-758$ \\
$759-761$ \\
$762-764$ \\
$765-778$ \\
779 \\
$780-782$ \\
$783-792$ \\
$793-808$ \\
$809-811$ \\
$812-822$ \\
$823-853$ \\
853 \\
$854-972$ \\
$903-902$ \\
$925-928$ \\
$929-936$
\end{tabular}


TI. Eetre

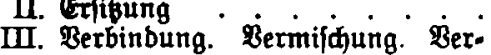
arbeitung

IV. Erverb von Erzeugnifien und Ion: ftigen Beftandteilen einer Eadje.

V. Îneignumg .

VI. Frunb . . . 256

23 ierter Titel. Injprüde aus bem

Eigentume. . . . . . . . 261

Fünfter Titel. MRiteigentunt: 268

Bierter gbjunitt. (Erbbau redt . . 270

Frinfter Yoidnitt. Dienft barleiten 271

Eriter Titel. Grunboienftbarleiten 271

3 we iter Titel. Riebbraud . . . 273

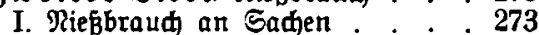

II. Rieв̈brauळ an Ređtten . . . . 285

III. Rieß̈braud an einem Bermögen 289

Dritter Titel. Bejuräntte perfönlidje Dienftbarłeiten

Eediter abinnitt. Bortaufìredt.294

Siebenter Abidnitt. Realla lten. 298

Foter Mbidnitt. gpothet. (B) $r u n b$.

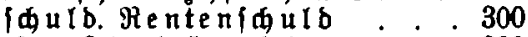

Erfter Titer. Sonpothel . . . 300

8weiter Titel. Brunbjauld. Benten: jaulb

I. Brunbífurs

II. Renteniqulb . . 335

Reunter Mbidutt. Pfandredt a

berogliden Saben und an Red ten

Eriter $\mathfrak{T}$ itel. Bfanbredt an beweg. liden Sadien

Broiter Titel. Pianbredt an Redten

Biertes Pud. Familtentedt.

Criter abidnitt. Bürgerlide Ehe. 366

Eriter Titel. Berlöbnis... 366 3 tweiter Titel. Eingehung ber Ege 367

\author{
88 \\ $946-952$ \\ 953-957 \\ $958-964$ \\ 965-984 \\ $985-1007$ \\ 1008-1011 \\ 1012-1017 \\ 1018-1029 \\ 1030-1067 \\ 1068-1084 \\ 1085-1089 \\ 1090-1093 \\ $1094-1104$ \\ 1105-1112 \\ $1113-1190$ \\ $1191-1198$ \\ 1199-1203 \\ $1273-1296$ \\ 1297-1302 \\ $1303-1322$
}

337

337 : 1204-1272 
Dritter Titel. Pidtigleit unb $\mathscr{M}=$ fectbarleit ber Eke. . . . . . 372 Bierter Titel. Wiederberheiratung im Falle ber Tobeşerflärung . . 378 Fünfter Titel. Mirfungen ber Ege im allgemeinen . . . . . . 382

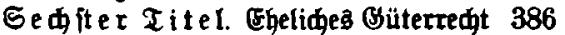
I. Beleßglides Büterredt . . . . $\mathbf{3 8 6}$

1. थllgemeine Roridriften . . 386

2. Berwaltung und Mußnięung 387

3. Sdjulbenhaitung . . . 395

4. Beendigung ber Berwaltung unb

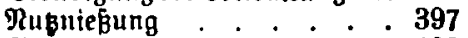

5. Bütertreınung . . . . . 402

II. Bertragämäß̆iges ßüterred̆ . . 404

1. MIlgemeine 23oridriften . . 404

2. Allgemeine Bütergemeinidaft . 405

3. Exrungenidaftsgemeinidaft .439

4. Fahruisgemeinihajt . . . 467

III. Büterredt'šrengifter . . . . . 469

Siebenter Titel. Edeibung ber Ehe 471 Adter Titel. Sirdlide Berpflidtungen . . . . . . . . 479

Bweiter Wbidnitt. Berwandtidait 479

(Eriter Titel Mllaemeine Boridriften 479

3 weiter Titel. Ehelide Ubftammung 479

Dritter Titel lunterhaltspflidt. 482

Bierter Titel. Redtlide Stellung ber eheliden Sinter . . . . . 487

I. Red)t şverhältnis z roifden ben (Eltern uno bem finbe in allgemeinen . 487

II. Elterliche Bemalt . . . . . 489

1. Eiterliche Bewalt bes Baters . 489

2. Elterlide Betwalt ber Mutter 510

$\mathfrak{F}$ ünfter Titel. Redtlidje Stellung ber Stinder aus niditigen Egen. . 516 Sediter Titel. Jiedtlidge Stellung ber uneheliden finder

$1348-1352$

$1353-1362$

$1363-1372$

$1373-1409$

$1410-1417$

$1418-1425$

$1426-1431$

$1432-1436$

$1437-1518$

$1519-1548$

$1549-1557$

$1558-1563$

$1564-1587$

1588

1589,1590

$1591-1600$

$1601-1615$

$1616-1625$ 1626

$1627-1683$

$1684-1698$

1699-1704

$1708-1718$ 

Siebenter $\mathfrak{T}$ it el. Regitimation un-
ehelider Finber.

I. Regitimation burd nadfolgenbe Ehe

II. EGheliditeitßerllärung .

Á) ter Titel. Annahme an Sinbes Statt

Selte

88

521

Dritter qbidnitt. Bormu

Erfter Titel. Bormunbidaft ïber Mrinberjährige

I. Trorbnung oer gormunbidait: $\mathbf{5 3 4}$

II. Führung ber Rormunbliaft .

II. Fubrung ber Bormunbidaft. .

III. Füriorge und Quffidit bes Bormunbinaftzgeridt 3

IV. Wittwirtung bes Bemeinbemailen: rat3 . . . . . . . . . $\mathbf{5 5 5}$

V. Befreite Bormuttbidaft . . . 55b

VI. Familientat . . . . . . . 558

VII. Beenbigung ber Bormunbiđaft. 565

Breiter Titel. Bormunbláft über

Bolliāahrige

Dritter Titel Pflegifaft $: .573$

$1719-1722$

523 1723-1740

$1741-1772$

534 $1773-1792$

$1793-1 \times 36$

551 | $1837-1848$

$1849-1851$

$1852-1857$

$1858-1881$

$1882-1895$

$1896-1908$

$1909-1921$

\section{Zitnites Pu西. Erbredt.}

Criter abfunitt. Erbfolge. . . 579

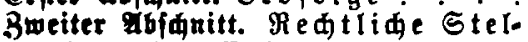
I $u$ g bes Ex $x$ en

Eriter Titel. Annahme und $\mathfrak{A u g}_{\text {. }}$

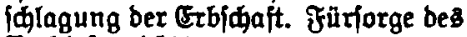
Radlásgetidts

3 weiter Titel. Şaitung bes Erben

für bie Naßlaßberbinblidleiten . 588

I. Radlab́berbinblidleiten . . . 588

II. Iufgebot ber Rađlaßgläubiger . 588

III. Bejürönfung ber saftung beş Erben

$1922-1941$

1V. ت̋nbentarerridhtung. Unbejhräntte Saftung bez Erben ... . .

$590 \quad 1975-1992$

V. Ouficiebende Cinreben - . . 504 1993-2013

596 1993-2013 604 2014-2017 
Dritter Titel. Erbíaftzaniprud 605

Bierter Titel. Mehrheit von Erben 610

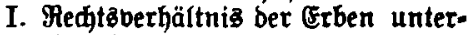
einanber

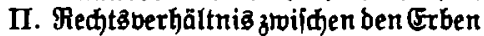
unb ben Tađhlapgläubigern . . 621

2032-2057

2058-2063

Dritter Tbfonitt. Teit a ment . . 6:3

Erfter Titel Allgemeine Borfdriften 623

3weiter Titel. Erbeinjebung . 628

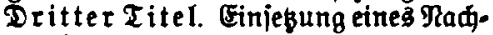
erben

Bierter Titel. 2kermähtnis . . 645

Fünfter Titel. Iuflage . . . 660

Ged fter Titel. Teitamentäbolliftreder 664

Siebenter Titel. Erridtung unb $\mathfrak{X} u$ f-

hebung eines̉ Teptamentä. . . 672

Ud ter Titel. Gemeinidaftlides Tejta= ment .

Bierter Tbidnitt. (E) buertrag. 683

Fanfter 9bidnitt. Pflidtteil . 696

Eedfiter grbinitt. E r buntoür big: teit

$2229-2264$

$2265-2273$

$2274-2302$

$2303-2338$

707

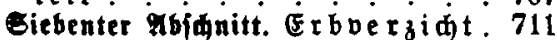

Fdter Fbidnitt. $E$ r b f

Reunter 9bidnitt. Frbidaft g tauf 714

$2339-2345$

$2346-23 \div 2$

$2353-2370$

2371-2385

\section{Cinführungsgeję zum Bürgerlthen Dejębudje.}

Erfter abiqnitt. Allgemeine Boriळriften

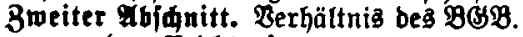
zu Den Meiđjgeieşen

\begin{tabular}{c|c} 
Eetie & Art. \\
721 & $1-31$ \\
729 & $32-54$ \\
& \\
743 & $55-152$ \\
789 & $153-218$
\end{tabular}

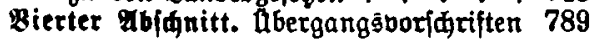

Gadregifter . . . . . . . . . 813 
